\title{
Essentials of Diabetes Care in Family Practice
}

\author{
Hakan Demirci, Ilhan Satman, Yıldırım Çınar and Nazan Bilgel
}

Additional information is available at the end of the chapter

http://dx.doi.org/10.5772/48616

\section{Introduction}

Worldwide there are a large number of patients with diabetes mellitus (DM) and the prevalence is increasing rapidly. About $20-30 \%$ of patients visiting family practice units suffer from DM or its complications. Family physicians require in-depth knowledge of DM. A family physician is expected to know about the disease as well as the drugs prescribed to treat the disease. They should provide practical and comprehensive care to DM patients. The diagnosis of a disease must be easy and less costly and should provide an estimation of the risks facing a patient in the future if precautions and recommendations are not adhered to. Family physicians need standard algorithms to follow their patients with DM. This chapter summarizes simple definitions, routine assessments, standard treatment and preventive options that should be known, easily recognized and applied by family physicians providing daily care to patients with DM.

\section{History}

Symptoms of DM have been reported since very old times. Diabetes is a word of Greek origin and means a "siphon". Aretus the Cappadocian, a Greek physician who lived in the $2^{\text {nd }}$ century, named the condition such because his patients had polyuria and passed water like a siphon. Mellitus is a Latin word and means "honey". The combination of these two words describes an illness that is associated with frequent and sweet urination. Current medical knowledge supports the ancient definition because it is now known that plasma glucose levels over $180-200 \mathrm{mg} / \mathrm{dL}$ impair glomerular filtration and pollakiuria and glycosuria are the result.

In 2009, the American Diabetes Association (ADA) and European Association for the Study of Diabetes (EASD) published a consensus report stating that the A1C can be used as a criterion for the diagnosis of DM [ADA, 2012]. Table 1 summarizes the current diagnosis approach. The A1C measure defines DM clearly for family physicians and 
prevents confusion in the diagnosis of patients with DM. There are some other well established tests, including the oral glucose tolerance test. However, it takes time to obtain a definitive result from this test as repeated blood samples (3-4 over 2-3 hours) are required to measure plasma glucose. In addition to diagnosis of $\mathrm{DM}$, the $\mathrm{A} 1 \mathrm{C}$ provides clues about the possible complications that the diabetic patients may be faced with. The $\mathrm{A} 1 \mathrm{C}$ is also used to direct treatment options. If the A1C values are within normal limits then a decision whether to continue with the ongoing treatment is made. This criterion has been the topic of much debate in Medical arena as many authorities do not agree with the use of A1C as a diagnostic criterion. It has been argued that the A1C may not able to detect DM characterized by postprandial hyperglycemia. Another argument relates to the standardization of the A1C measurement techniques used by laboratories. Therefore, a standardized method needs to be used, and it is recommended that the A1C should be confirmed with fasting plasma glucose (FPG $\geq 126 \mathrm{mg} / \mathrm{dL}$ ). However, laboratories that serve family physicians are not always set up to measure the A1C. In addition, the A1C values may vary depending on race and ethnicity [Ziemer et al. 2010 \& Kumar et al. 2010].

\begin{tabular}{|c|c|c|c|c|}
\hline & FPG & $\begin{array}{l}\text { OGTT 2-h } \\
\text { PG }\end{array}$ & Random PG & $\mathrm{A} 1 \mathrm{C}$ \\
\hline $\begin{array}{l}\text { Diabetes } \\
\text { Mellitus }\end{array}$ & $\begin{array}{l}\geq 126 \mathrm{mg} / \mathrm{dL} \\
(7.0 \mathrm{mmol} / \mathrm{L})\end{array}$ & $\begin{array}{c}\geq 200 \mathrm{mg} / \mathrm{dl} \\
\quad(11.1 \\
\mathrm{mmol} / \mathrm{L})\end{array}$ & $\begin{array}{c}\geq 200 \mathrm{mg} / \mathrm{dl} \\
(11.1 \mathrm{mmol} / \mathrm{L}) \\
\text { and classic } \\
\text { symptoms of } \\
\text { hyperglycemia }\end{array}$ & $\begin{array}{c}\geq 6.5 \% \\
(\geq 48 \mathrm{mmol} / \mathrm{mol})\end{array}$ \\
\hline $\begin{array}{l}\text { Increased risk } \\
\text { for DM } \\
\text { (prediabetes) }\end{array}$ & $\begin{array}{c}100-125 \\
\mathrm{mg} / \mathrm{dL} \\
(5.6-6.9 \\
\mathrm{mmol} / \mathrm{L})\end{array}$ & $\begin{array}{c}140-199 \\
\mathrm{mg} / \mathrm{dL} \\
(7.8-11 \\
\mathrm{mmol} / \mathrm{L}) \\
\end{array}$ & & $\begin{array}{c}5.7-6.4 \% \\
(39-46 \mathrm{mmol} / \mathrm{mol})\end{array}$ \\
\hline
\end{tabular}

*[ADA, 2012]

Table 1. Current criteria for diabetes mellitus diagnosis and increased risk for diabetes mellitus*

Over the last decade we have observed some major modifications in the treatment options related to DM. In 2006, the ADA reported that life-style changes alone are not sufficient to overcome DM in individuals recently diagnosed with the disease. Based on meta-analyses studies, Metformin has been included as a first line treatment option, if no contraindications are present [Nathan et al., 2006]. Metformin has many beneficial effects in the treatment of DM and it is very cost effective. Metformin decrease plasma glucose (PG) and has been shown to slightly alleviate obesity and reduce elevated blood lipids. However, it is well known that metformin is not always well tolerated and therefore half the target dose is recommended during the initial stages of metformin therapy. Metformin therapy is not recommended for patients with creatinine levels exceeding the upper limit of normal range $(>1.4 \mathrm{mg} / \mathrm{dL})$ or if eGFR less than 60 $\mathrm{mL} / \mathrm{minute}$. 
The ADA, supported by the EASD, indicated that a global target for reasonable glycemic control should be A1C $<7 \%$. However, International Diabetes Federation (IDF), the local societies, like Society of Endocrinology and Metabolism of Turkey (SEMT) and others suggested a slightly lower target (A1CA1C $\leq 6.5 \%$ ) [ADA, 2012; IDF, 2005; SEMT, 2011]. However, from 2010, individual specifications such as diabetes duration, life expectancy, and previous glycemic control status, associated co-morbidities, and complications are advised to be taken into account before setting the target of glycemic control.

\section{Pathogenesis}

Diabetes Mellitus is characterized by hyperglycemia that results from defects in the secretion and/or action of insulin. DM is primarily classified into two groups, type $1 \mathrm{DM}$ and type $2 \mathrm{DM}$. Generally, type $1 \mathrm{DM}$ is accepted as an autoimmune disease with some triggering factors usually responsible for the development of autoimmunity. Destruction of pancreatic $\beta$-cells by several autoantibodies (i.e. islet cell cytoplasmic antibodies; ICA, antibodies against glutamic acid decarboxylase; Anti-GAD, islet associated or anti-tyrosine phosphatase antibodies; IA2, anti-fogrin antibodies; IA2- $\beta$, insulin autoantibodies; IAA, and antibodies against zinc transporter-8; Anti-ZnT8) is the typical explanation regarding the mechanism for the development of type $1 \mathrm{DM}$. On the other hand, peripheral insulin resistance, loss of $\beta$-cell reserve or $\beta$-cell exhaustion, problems in hepatic glucose production, abnormalities in glucose absorption and obesity are the main pathological characteristics of type 2 DM [Barnet \& Braunstein, 2007].

\section{Epidemiology}

$\mathrm{DM}$ is a major epidemic of the $21^{\text {st }}$ century. However, reports published in 2010 demonstrated that the estimated targets for 2025 have already been reached, 15-20 years earlier than expected. According to the recent estimates by IDF (IDF Fifth Atlas, 2011), there are 366 million people with DM worldwide, and the population with DM is expected to increase to 552 million by the year 2030 .

It has been shown that the risk for DM is higher in individuals who have not completed formal education as compared with those who received a high school or university education. In cross-sectional studies current smoking has been suggested to have a protective effect that may be explained by lower caloric intake or weight loss associated with smoking. However, ex-smokers are more prone to developing DM compared with nonsmokers due rapid weight gain [Amorosa et al., 2011]. Recent guidelines decreased the diagnostic level for FPG and this increased the number of people diagnosed with DM. In addition, a sedentary life-style, poor diet, obesity and the lack of regular physical activity contributed to reaching the 2030 DM estimates early [Satman et al., 2010]. The increased prevalence of childhood obesity has been associated with insulin resistance. For children born after the year 2000 there is a $32.8 \%$ risk of developing DM in males and $38.5 \%$ risk in females [Amorosa et al., 2011]. 


\section{Routine assessments}

Routine assessments are very important in DM because these allow physicians to monitor the development of the disease and associated complications. These assessments provide early identification of co-morbidity, risk factors as well as complications of DM that include coronary artery disease (CAD), impotence, neuropathy, nephropathy, retinopathy and blindness. The combination of neuropathy, retinopathy and nephropathy in DM is termed the "triopathy". Awareness of retinopathy is typically found in individuals who have had a family member who experienced the same complication or those actively seeking information relating to the complications of DM. Family doctors should inform their patients about the danger of complications. They should ensure that the appropriate laboratory examinations are performed in time and refer patients to specialists or colleagues if required. A plan for the management of DM should be developed and maintained between the physician and the patient. Compliance of the patient to the plan should be monitored by the physician.

\subsection{Glycosylated hemoglobin A1C}

The A1C percentage may reflect average glycemic control over several months [Sacks et al., 2002]. The A1C test helps in the diagnosis of DM and is also valuable for the determining the efficacy of treatment. In addition, the test is useful for determining DM complications (Table2).

As we mentioned previously, A1C target levels differ from patient to patient. For the elderly and children the aim is not to have a tight control of the A1C level. Tight control may disturb quality of life of the patient and it may increase health risks. Immaturity in children $<6$ or 7 years and their inability to recognize hypoglycemia leads to modifications in glycemic goals. There is small data available to show benefits of intensive glycemic, blood pressure, and lipid control in the elderly [ADA, 2012].

\begin{tabular}{|c|c|}
\hline & Retinopathy 35\% $\downarrow$ \\
Type 1 DM (DCCT study) & Neuropathy 30\% $\downarrow$ \\
& Nephropathy $24-44 \% \downarrow$ \\
\hline & Micro vascular complications $35 \% \downarrow$ \\
Type 2 DM (UKPDS study) & Death rate in DM $25 \% \downarrow$ \\
& MI 18\% $\downarrow$ \\
& Mortality of all causes $7 \% \downarrow$ \\
\hline DM, diabetes mellitus; DCCT, Diabetes Control and Complication Trial; UKPDS, United \\
Kingdom prospective Diabetes Study; MI, myocardial infarction.
\end{tabular}

Table 2. Relationship between complications and 1\% decrease in A1C

\subsection{Microalbuminuria and estimated glomerular filtration rate (eGFR)}

Microalbuminuria measurements can be performed using different methods. Albumin and creatinine determination in spot (preferably first morning) urine sample is preferred to 24 
hour urine analyses [Eknoyan et al., 2003, Levey et al., 2003]. Determination of early morning microalbuminuria in spot urine is an easy method that can be obtained in a family practice setting but a limitation is that this method is associated with false positives. In addition, hydration status affects the test results. However, albumin to creatinine ratio $(\mathrm{ACR})$ is less affected by dehydration. Classification of microalbuminuria state is shown in table 3 .

Diagnosis of persistent microalbuminuria should occur if positive in two out of three urine samples over six months. It should be remembered that exercise in the previous 24 hours, infections, congestive heart failure and uncontrolled hypertension may interfere with the results. The ADA and EASD recommend microalbuminuria testing at least once a year.

\begin{tabular}{|l|l|}
\hline Normal & $<30$ \\
\hline Microalbuminuria & $30-299$ \\
\hline Macroalbuminuria & $\geq 300$ \\
\hline
\end{tabular}

Table 3. Microalbuminuria (ACR; early morning spot urine albumin $[\mu \mathrm{g}] /$ creatinine $[\mathrm{g}]$ )

Estimated glomerular filtration rate (eGFR) is another method for evaluating renal function. The Modification of Diet in Renal Disease (MDRD) formula can be used to estimate GFR. There are websites for calculating eGFR on the internet. Some laboratories note the results of eGFR together with creatinine measurements [Levey et al., 1999]. eGFR $<90 \mathrm{~mL} / \mathrm{minute}$ indicates renal failure.

\subsection{Serum cholesterol}

"Diabetic dyslipidemia" is considered in individuals with low HDL-cholesterol and high TG. The targets for HDL-cholesterol should be $\geq 50$ for females and $\geq 40 \mathrm{mg} / \mathrm{dL}$ for females, and $<150 \mathrm{mg} / \mathrm{dL}$ for TG. Hyperlipidemia is a common co-morbidity seen in DM. The Framingham Heart Study found that DM and hyperlipidemia are major risk factors for the development of coronary artery disease. Lipid profiles should be checked every year in patients with DM. Precautions should be taken if there is an elevated lipid profile. Low density lipoprotein cholesterol (LDL-cholesterol) levels $<100 \mathrm{mg} / \mathrm{dL}$ must be achieved as soon as possible. If there is already a history of coronary artery event then the target for LDL-cholesterol should be revised to $<70 \mathrm{mg} / \mathrm{dL}$ [Grundy et al., 2004].

\subsection{Fundus examination}

Screening for diabetic retinopathy gained importance after reports that laser photocoagulation was very effective in preventing visual loss (DRS Group, 1976). Fundus examination by an ophthalmologist at least once in a year is recommended. Some family physicians may have experience examining the retina; however it would be better to do so in a hospital setting. Examination can be performed using an ophthalmoscope or a slit lamp. Fluorescein angiography (FFA) can be used to determine the location of the leakage in suspected cases. 
Diabetic retinopathy is classified as non-proliferative or proliferative in nature. Once a retinal hemorrhage has been identified it is recommended, especially for individuals with proliferative retinopathy, the assessment should be performed more frequently.

\subsection{Foot examination}

Physicians should examine the feet of DM patients preferably in every visit to help prevent the development of the "diabetic foot ulcers". A careful inspection and palpation of lower extremity pulses is required. Skin temperature must be checked and increases or decreases in the regular temperature, suggests the existence of an underlying problem. Skin hair loss, atrophy, cracked skin, or poor circulation suggests neurotropic or autonomic changes. Cracked skin is a haven for infections and a loss of circulation delays wound healing. Patients should be educated about these changes. If they notice something abnormal they should inform their family physicians about the problem.

\section{Microvascular complications}

Microvascular complications of DM worsen the quality of life and cause increased morbidity in patients. These complications include diabetic foot ulcers that family physicians should be aware of as well as prevent their progression. In the case of DM related end stage renal disease (ESRD), hemodialysis is usually considered. This situation also worsens the quality of life for patients with DM. Diabetic retinopathy is the most common cause of blindness. However, before reaching the stage of blindness it causes occupational and some other serious problems. This in turn affects other members of the family if the patient was the only one who works outside. Considering complications in DM it should be kept in mind that the first thing to do is to control PG levels and regulate hypertension if it is present. This approach will not only prevent most of the complications but also stops the progression of the pre-existing complications.

\subsection{Retinopathy}

Excluding traumatic cases, DM is the most common cause of blindness. During the first two decades of DM, nearly all the patients with type $1 \mathrm{DM}$ and more than half of the patients with type 2 DM experience retinopathy to some degree [Budak et al., 2004].

It is possible to diagnose retinopathy while performing routine ophthalmologic examination but family doctors should suspect diabetic retinopathy when microalbuminuria is detected in the urine. In addition, patients with known CAD have a greater risk of retinopathy.

Early detection of retinopathy should be one of the priorities when assessing DM patients. Early detection ensures a beneficial response to treatment. A FFA verifies the suspected retinopathy and photocoagulation is the preferred treatment if the diagnosis is evident. Ophthalmologists attempt to coagulate leaking retinal vessels to prevent the progression of retinal edema. Improvement in DM retinopathy is associated with reduced PG regulation [Klein (1995)] and blood pressure [Leske et al., 2005]. 


\subsection{Neuropathy}

Neuropathy is one of the most common chronic complications of DM (the prevalence over 20 years duration of DM is approximately 70\%) but is usually under diagnosed [Barnett \& Braunstein 2007]. Duration of DM and poor PG control correlates with the occurrence of diabetic neuropathy. Peripheral neuropathy is seen in $30 \%$ of cases. Neuropathy of nerves in the feet provides the earliest symptoms. Neuropathy has been sub-divided into three groups (autonomic, focal, and diffuse). Autonomic neuropathy has three components, cardiovascular, gastrointestinal and genitourinary. Examples of focal neuropathies are mononeuritis and carpal tunnel syndrome. Proximal motor neuropathy and distal symmetric polyneuropathies are diffuse neuropathies. Paresthesia can be described as numbness, burning or pins and needles in extremities. A characteristic of paresthesia is that the pain increases at night [Amorosa et al., 2011].

Analgesics, antiepileptic drugs and antidepressants can be used in the treatment of painful neuropathies. Amitriptilin, an antidepressant drug, has been used widely for pain relief in painful neuropathic cases. Anticonvulsants such as gabapentin, and pregabalin or SSRIs such as duloxetine, are modern pain relief drugs commonly used in painful neuropathies. B complex vitamins have also been used to treat neuropathy; however recent studies do not support their use. It has been suggested that long term metformin use may cause iatrogenic neuropathy due to malabsorption of vitamin B12 [Attarian 2011].

\subsection{Diabetic foot ulcers}

Diabetic foot ulcers are due to impaired nerve innervations in the lower extremities. This in turn causes decreased circulation that result in deficient migration of leukocytes to infected areas. Dryness of the extremity causes cracked skin that increases the risk of microorganisms settling there. Insufficient immune responses prevent local inflammation and subsequent healing.

Management of mild to moderate cases of diabetic foot should be provided by a foot care team including a physician, vascular surgeon, orthopedist, plastic surgeon, podiatrist, nutritionist, and physiotherapist. Treatment of advanced diabetic foot ulcers requires long hospitalization periods, and is costly. Treatment often requires surgery and in some cases the amputation of the affected finger or foot. In certain situations, an amputation or revised amputation at a higher level is indicated. Hyperbaric oxygen therapy is considered an adjuvant therapy and may be effective in some cases [Cimşit et al., 2009]. This therapy enables accelerated circulation to hypoxic, infected or ulcerated parts of the foot. Anemia should also be corrected as soon as possible to enhance wound healing. A neglected, small injury may cause the loss of a finger, foot or a leg.

The basic approach in DM patients suffering from any complication is prevention. Family physicians stand at the center of treatment. They should educate their patients about foot care and inform them about importance of PG regulation and advise the cessation of 
smoking. Patient education also includes information and training about topics such as foot inspection, appropriate shoes and socks, nail cutting and the importance of consulting a podiatrist [Barnett \& Braunstein 2007].

\subsection{Nephropathy}

The incidence of DM related nephropathy is 20-30\% [Barnett \& Braunstein 2007]. Microalbuminuria and eGFR should be measured every year in patients with DM. When macroalbuminuria is diagnosed then a protein restriction diet is recommended. Blocking the renin-angiotensin system (RAS) by drugs such as ACE inhibitor or ARB is advised to reduce BP, established microalbuminuria and CAD outcomes [HOPE study group 2000]. ESRD is the undesired complication if the recommended treatment does not work. ESRD is usually treated by hemodialysis. Nephropathy of different stages is not rare in a population, but it is often undiagnosed. It is possible to evaluate eGFR if serum creatinine is measured. The eGFR results can be used effectively by family physicians for preventing the progression to renal failure. Hemodialysis on top of DM can make the life more difficult for diabetic patients.

\subsection{Erectile dysfunction}

Erectile dysfunction affects over $50 \%$ of men with diabetes $>60$ years old. Its etiology is multifactorial. It was shown that erectile dysfunction may be a signal for silent CAD [Phe \& Rhoupret 2012]. Sildenafil may be effective in its treatment. However, risk of accompanying cardiovascular problems limits sildenafil use. Cessation of smoking should be strongly recommended.

\section{Macrovascular complications}

Accelerated atherosclerosis causes macrovascular complications that include CAD, cerebral and peripheral vascular diseases. It is not unusual for DM patients to experience a stroke more than once. Macrovascular complications are the primary cause of death in patients with DM.

\subsection{Hypertension}

When considering macrovascular complications, controlling hypertension is even more important than the regulation of hyperglycemia. ACE inhibitors are the first line of therapy, despite the finding that ARB and calcium channel blocker drugs may be as effective. The target for the reduction of blood pressure is slightly lower than normal (systolic $<130$ and diastolic $<80 \mathrm{mmHg}$ ). Studies have shown that blood pressure over $115 / 75 \mathrm{mmHg}$ carries a high risk for CAD [Chobanian et al., 2003].

On average about three different types of drugs are needed to decrease blood pressure in patients with DM. 


\subsection{Coronary artery disease}

The most important complication related to mortality and morbidity in DM is CAD. DM is considered a cardiovascular disease equivalent. Major CAD risk factors should be evaluated once a year and identified risks (e.g. hyperlipidemia, hypertension, smoking and microalbuminuria) should be treated. In general, asymptomatic patients and/or patients with a "normal" resting electrocardiogram (ECG) are not recommended for further screening for CAD. However, DM patients who have not been diagnosed with CAD are considered to have the same risk as non-diabetic CAD patients [Barnett \& Braunstein 2007]. $\mathrm{DM}$ patients diagnosed with CAD risk are recommended to use aspirin. However, based on recent meta-analyses aspirin is not routinely recommended for primary prevention of CAD. ACE inhibitors and statins should be added to therapy for DM patients with CAD. Post Myocardial Infarction (MI) patients are advised to use a $\beta$-blocker for at least two years if tolerable.

\subsection{Stroke}

About $80 \%$ of patients with DM die from stroke or CAD. During a stroke blood supply to the brain is reduced resulting in brain tissue damage. Sudden onset paralysis, cognitive and speech impairments are observed. Clot dissolving treatment must be started as soon as possible. Stroke prevention and treatment requires the reduction of lipids and hypertension. Cessation of smoking is mandatory.

Usually it is not advised to decrease PG levels too quickly especially if long-term poor glycemic control exists.

\section{Treatment}

Treatment of DM is multidisciplinary and unique for every patient. The first step of treatment in DM is acceptance of the disease. Every patient should receive comprehensive information about the seriousness of the disease. Importance of self-care in DM must be stressed. Establishment of confidence between the physician and patient should be established before treatment algorithms are implemented. The patient should accept that with the help of the family physician the disease can be controlled. During face to face interviews patients must be made aware that the disease is chronic and that treatment will be life-long. It should be emphasized that if precautions are not taken this will increase the risk of complications that may increase morbidity and mortality. The patient should be informed that DM may affect their quality of life as well as that of their family. On the other hand, the patient should also be made aware that DM is a common condition. Its prevalence is assumed to be more than $10 \%$ worldwide and this means that the patient is not alone. The above general considerations regarding acceptance of the disease takes time, patience and experience.

The conventional treatment guidelines recommend metformin administration as soon as the diagnosis has been established. Metformin therapy is cost effective. However, for cases 
where the A1C values $>10 \%$ and/or FPG $>300 \mathrm{mg} / \mathrm{dL}$ and/or complaining about severe hyperglycemic symptoms then insulin therapy must be commenced immediately. Recently, a common approach has been to use a long acting insulin analog injection once daily with or without an oral antidiabetic drug.

Persuading a patient to transfer to the use of insulin is difficult and sometimes impossible. The public perception of insulin therapy is poor. Insulin is considered the end of the road and some individuals see it as a "poison".

In medicine the existence of treatment "fads" or "myths" is ever present. Over-time the truth we believe in may turn out to be a false. Physicians should work with evidence-based science. Family physicians must be up to date with current DM treatment practices. Insulin is the accepted drug used in the treatment of DM. It is claimed that high dose insulins such as glargine or NPH, and pioglitazone may be associated with slightly increased risk of some kinds of malignancies. Researchers have not been able to link between the development of breast cancer and glargine insulin or between bladder cancer cases and pioglitazone. Both of these medications are very successful treatment options.

In daily practice it is not surprising to observe patients that are using two or more different kinds of oral antidiabetic drugs despite this not being recommended in the treatment guidelines. Unfortunately, patients sometimes use drugs that have the same mechanism of action. This may result in hypoglycemia. It is not possible for family physicians to know every disease and every drug. However, DM is a common chronic disease and therefore family physicians should have comprehensive knowledge of the medication used for all stage of the disease. This will increase the physician's confidence and increase the level of confidence between the patient and the physician.

Resistance to insulin can be classified into three types: Clinical insulin resistance, patient's resistance to insulin injection and physician's resistance to prescribe insulin. Of these the first type is the true biological resistance that plays a role in the development of DM. Physicians who are not confident in prescribing insulin therapy should refer their patients to an experienced specialist. Any delay in referral may result in harm to the patient and an increased risk of developing complications. Research has shown that a $1 \%$ drops in the A1C will result in a $21 \%$ decrease in complications and decrease mortality and morbidity rates in DM patients. Keeping patients unaware of suitable therapy such as insulin is not ethical and may be considered as malpractice. Hospitalization is not a rule when transferring to insulin. If the patient is not critically ill then education about insulin injections can be given at the family practice. A physician and/or a nurse trained that has received training in DM care can educate the patient.

It is a common approach to start injecting basal insulin using a dose of 0.2 Units/day. If the insulin is a premixed human short acting and long acting then $2 / 3$ of the total dose is injected in the morning and $1 / 3$ in the evening. Human insulin mixtures are preferably injected 15-30 minutes before the main meal but insulin analogs are injected just 5-15 minutes before the meal. If a mixture of analog insulins is prescribed, then about $50-60 \%$ can be given in the morning, and the rest before dinner. Patients should self-monitor their 
glucose levels and share the results with their physician. Postprandial measures are obtained at the end of the second hour following the meal. At the start of treatment, if there are no extraordinary problems, the physician evaluates the self monitored results every three days and based on these results decides on how to manage the optimal dose of insulin.

Insulin injections are administered using a special pen that can be adjusted to comfortably inject the desired doses. Education on the use of the pen should be provided to the DM patients. Family physicians should instruct patients to keep insulin in refrigerator at an optimal temperature and not to allow it to freeze. Injection methods should be explained and demonstrated in person. A description regarding the use of the insulin pen is provided in the pen boxes. However, this may confuse beginners as well as individuals who are scared of injections. During the insulin education interview patients may be asked to write down the steps one by one in their own words so that at the time of application there is no confusion. It is known that insulin absorption is faster around the umbilicus where there is a large amount of vessels.

Patients must be informed about hypoglycemia. Hypoglycemia usually occurs in response to fasting for a long time and/or when individuals perform with extra effort compared with normal. Signs of hypoglycemia are cold sweating, tachycardia, blurred vision and changes in consciousness. If the physician informs patients about the risk of hypoglycemia before they experience it, this may help in the management of hypoglycemia treatment, improving trust between the patient and physician. In addition, a better compliance to the treatment will be achieved.

Attempting to determine the optimal insulin dose as soon as possible is not recommended. In cases of stroke and retinopathy, smooth increases in insulin doses are advised. Target A1C levels may differ in different groups. For example, for the children and the elderly, targets are not as low as in adults. Diabetes treatment should not be based on a single individual. It must be acknowledged that the diagnosis of DM in a small baby will obviously affect the whole family. Sometimes a diabetic woman may be forced to give up insulin injections by her husband. If this is the case, family physicians must provide extra attention to these patients. Care in DM needs to be directed not only to the patient but also to the family and at times, the public.

\section{Noninsulin therapy (drugs)}

\subsection{Insulin sensitizers: Biguanids (metformin)}

This medication is recommended as first line therapy in addition to life style modifications. It is important to check creatinine levels when using the drug. A creatinine level that is $>1.4$ $\mathrm{mg} / \mathrm{dL}$, or an eGFR $>60 \mathrm{~mL} /$ minute is critical when making a decision to discontinue the drug. When the drug is used for the first time by a patient a stepwise manner is recommended. Half or sometimes one quarter of the dose is started for 3-7 days and then increased gradually until the total dose is administered. Gastrointestinal symptoms are common if the drug has not been taken 1-2 hours after a meal. Metformin may have a mild positive effect on weight loss and is therefore its use is preferred in obese individuals. 


\subsection{Insulin secretagogues: Sulfonylureas (gliclazide)}

Gliclazide is an alternative group of oral antidiabetic drugs. The risk of hypoglycemia is the major adverse effect and it may last more than 24 hours. It is usually preferred in patients older than 30 years old, who are relatively thin and have had DM for no longer than 5 years.

\section{Others:}

$\alpha$-Glucosidase inhibitors (acarbose)

It has mechanism of action on glucose absorption from gastrointestinal tract. Gastrointestinal side effects like flatulence, abdominal discomfort and diarrhea have been observed. In elderly it is preferred because there are no serious side effects like hypoglycemia when the drug is used alone.

\section{DPP-4 inhibitors (Sitagliptin)}

These are known as insulin like oral glucose-lowering drugs. They selectively and reversibly block degradation of GLP-1 and other incretins. They promote insulin sensitivity and improve $\beta$-cell function.

\section{Insulin therapy}

\subsection{Long acting insulin}

NPH insulin, Glargine insulin and detemir insulin are classified as long acting insulin. The first two are recommended once a day but detemir insulin can be used twice a day. If the patient is recommended to use insulin once a day, depending on the patient's reaction, it can be injected in the morning or in the evening. To avoid hypoglycemia while sleeping the insulin can be injected in the evening but sometimes it works better when injected in the morning.

\subsection{Short acting insulin}

Short acting insulin is primarily used as a part of intensive insulin therapy. Usually it is administered three times a day before the meals. Insulin analogues can be injected just before the meal.

\subsection{Mixed insulin}

There are different formulations for insulin mixtures. Probably the most commonly used formulation is the 30/70 insulin mix that is composed of $30 \%$ short acting and $70 \%$ long acting insulin. It is advised that the insulin is mixed well before use. In general, $2 / 3$ of the total daily dose is injected before breakfast and the remaining $1 / 3$ before dinner. The reason why it is important to follow this protocol is because during the daytime the patient continues to eat but after dinner there may only be a small snack eaten just prior to sleeping. 
Therefore, to prevent hypoglycemia during the sleep a smaller dose is injected at dinner. There are also 50/50, 25/75 and 20/80 combinations. Preferably, the 20/80insulin mix is administered in the elderly while the 50/50 combination may be beneficial in diabetic patients who perform heavy work as well as individuals who have poor dietary habits. In these individuals the mix is injected three times a day.

\subsection{Intensive insulin therapy}

Intensive insulin therapy is indicated on some occasions. Probably the most effective method for injecting insulin injection is dividing the total dose into 4-5 administrations. In practice this is not comfortable. Physicians usually prefer to divide the total dose of insulin into 4 or more administrations when it exceeds 100 Units per day. It is recommended that family physicians consult with endocrinologists when intensive insulin therapy is considered.

\section{Gestational diabetes mellitus}

Gestational diabetes mellitus (GDM) is diagnosed during the pregnancy and may sometimes persist after the baby is born. Family physicians should screen for GDM as soon as possible. Screening for GMD is recommended in the general population if risks for diabetes are present. If there is no DM risk in the pregnant women a75 $\mathrm{g}$ oral glucose tolerance test is recommended at $24-28$ weeks of gestation. Following labor checking for the persistence of DM is recommended.

GDM carries risks for the mother and baby. Treatment options during the pregnancy are limited.

If GDM cannot be controlled by diet then insulin administration must be considered. Oral antidiabetic drugs may be teratogenic. Conventional insulin should be selected for treatment because there are not enough clinical data regarding the use of modern insulin analogs.

\section{Immunization}

The Centers of Disease Control and Prevention (CDC) recommend both influenza and pneumococcal vaccines for all patients with DM. The ADA also recommends that DM patients should be vaccinated against influenza and pneumonia because they are more susceptible to these diseases and are typically hospitalized for a longer time than nondiabetics and have an increased risk of dying. Therefore, the World Health Organization has a target to increase the number of DM patients that receive vaccinations to $75 \%$ against influenza and $50 \%$ against pneumonia.

\subsection{Influenza vaccine}

Hospitalization due to influenza is three times more common among diabetics who have not been vaccinated [Colquhoun et al., 1997]. Hospitalization in intensive care units is four times 
more likely in unvaccinated cases. Trivalent influenza vaccine is available and contains the expected subgroups of influenza in the region for a particular year. A history of anaphylactic egg allergy is a contraindication for influenza vaccines. Children under 36 months are vaccinated with an infant dose that is half the normal dose. If a child is less than nine years old and is being is vaccinated for the first time a vaccination is repeated one month after the initial vaccination. It is recommended that family members of diabetics are also vaccinated.

\subsection{Pneumococcal pneumonia vaccine}

Pneumococcal pneumonia is an important cause of morbidity and mortality in patients with DM. Patients over 65 years and who had an additional pulmonary or cardiovascular problem are at an increased risk. This vaccine is recommended every five years. Half of the patients may suffer from local irritation at injection sites. The vaccine can be injected at the same time as other vaccines (e.g. influenza vaccine) but preferably in the other deltoid muscle.

\subsection{Hepatitis B vaccine}

Currently, there are studies examining recommendations for Hepatitis B vaccinations in DM. The Advisory Committee on Immunization Practices (ACIP) recommended that all of the unvaccinated adults aged 19 through 59 years with DM be vaccinated against hepatitis $B$ [CDC 2011].

\section{Conflicts of interest}

The authors state that there are no conflicts of interest.

\section{Author details}

Hakan Demirci

Department of Family Medicine, Sevket Yilmaz Training \& Research Hospital, Bursa, Turkey

Ilhan Satman

Division of Endocrinology and Metabolism, Department of Internal Medicine, Istanbul Faculty of Medicine, Istanbul University, Istanbul, Turkey

Yildırım Çınar

Department of Internal Medicine, Trakya University, Edirne, Turkey

Nazan Bilgel

Department of Family Medicine, Uludag University, Bursa, Turkey

\section{Acknowledgement}

The authors express their thanks to Mrs Nazlı Demirci (philologist) for her support in editing the English language of this chapter. 


\section{References}

American Diabetes Association. (2012). Diagnosis and classification of diabetes mellitus. Diabetes Care. Vol. 35, No. 1, pp. 64-71.

Amorosa LF, Lee EJ, Swee DE (2011). Diabetes Mellitus. Rakel \& Rakel Textbook of Family Medicine $8^{\text {th }}$ Edition. Chapter 34, pp. 731-755.

Attarian S. (2011). Original articles on axonal neuropathy. Rev Neurol (Paris). Vol. 167, pp. 951-954.

Barnett PS, Braunstein GD. (2007). Diabetes Mellitus. Andreoli and Carpenter's Cecil Essentials of medicine $7^{\text {th }}$ Edition. Chapter 68, pp. 676-707.

Budak Y, Demirci H, Akdogan M, Yavuz D. (2004). Erythrocyte membrane anionic charge in type 2 diabetic patients with retinopathy. BMC Ophthalmology http://www.biomedcentral.com/1471-2415/4/14. Accessed 2004 Oct.

Centers of Disease Control and Prevention (CDC). (2011). Use of hepatitis B vaccination for adults with diabetes mellitus: recommendations of the Advisory Committee on Immunization Practices (ACIP). MMWR Morb Mortal Wkly Rep. Vol. 60 No.50, pp. 1709-11.

Cimşit M, Uzun G, Yildiz S. (2009) Hyperbaric oxygen therapy as an anti-infective agent. Expert Rev Anti Infect Ther. Vol. 7, No. 8, pp. 1015-1028.

Chobanian AV, Bakris GL, Black HR, et al. (2003). National Heart, Lung, and Blood Institute Joint National Committee on Prevention, Detection, Evaluation, and Treatment of High Blood Pressure; National High Blood Pressure Education Program Coordinating Committee. The Seventh Report of the Joint National Committee on Prevention, Detection, Evaluation, and Treatment of High Blood Pressure: the JNC 7 report. JAMA. Vol. 289, pp.2560-2572.

Colquhoun AJ, Nicholson KG, Botha JL, Raymond NT. (1997). Effectiveness of influenza vaccine in reducing hospital admissions in people with diabetes. Epidemiol Infect. Vol. 119, pp.335-341.

Eknoyan G, Hostetter T, Bakris GL, et al. (2003). Proteinuria and other markers of chronic kidney disease: a position statement of the national kidney foundation (NKF) and the national institute of diabetes and digestive and kidney diseases (NIDDK). Am J Kidney Dis. Vol. 42, pp. 617-622.

Grundy SM, Cleeman JI, Merz CN et al. (2004). National Heart, Lung, and Blood Institute; American College of Cardiology Foundation; American Heart Association. Implications of recent clinical trials for the National Cholesterol Education Program Adult Treatment Panel III guidelines. Circulation. Vol. 110, pp. 227-239.

Heart Outcomes Prevention Evaluation Study Investigators. (2000). Effects of ramipril on cardiovascular and microvascular outcomes in people with diabetes mellitus: results of the HOPE study and MICRO-HOPE sub study. Lancet. Vol. 355, pp. 253-259.

Klein R. (1995). Hyperglycemia and microvascular and macrovascular disease in diabetes. Diabetes Care. Vol. 18, pp. 258-268 
Kumar PR, Bhansali A, Ravikiran M. et al. (2010) Utility of glycated hemoglobin in diagnosing type 2 diabetes mellitus: a community-based study. J Clin Endocrinol Metab. Vol. 95, pp. 2832-2835.

Leske MC, Wu SY, Hennis A, et al. Barbados Eye Study Group. (2005) Hyperglycemia, blood pressure, and the 9-year incidence of diabetic retinopathy: the Barbados Eye Studies. Ophthalmology. Vol. 112, pp.799-805.

Nathan DM, Buse JB, Davidson MB et al. (2006) Management of hyperglycemia in type 2 diabetes: A consensus algorithm for the initiation and adjustment of therapy: a consensus statement from ADA and EASD. Diabetes Care. Vol. 29, pp.1963-1972.

Levey AS, Coresh J, Balk E, et al. (2003). National Kidney Foundation. National Kidney Foundation practice guidelines for chronic kidney disease: evaluation, classification, and stratification. Ann Intern Med. Vol. 139, pp. 137-147

Levey AS, Bosch JP, Lewis JB, Greene T, Rogers N, Roth D. (1999). Modification of Diet in Renal Disease Study Group. A more accurate method to estimate glomerular filtration rate from serum creatinine: a new prediction equation. Ann Intern Med. Vol. 130, pp.461-470.

Satman I and TURDEP II study group report (2011). 48. Ulusal Diyabet Kongresi, Antalya.

Sacks DB, Bruns DE, Goldstein DE, Maclaren NK, McDonald JM, Parrott M. (2002). Guidelines and recommendations for laboratory analysis in the diagnosis and management of diabetes mellitus. Clin Chem. Vol. 48, pp. 436-472.

The Diabetic Retinopathy Study Research Group. (1976). Preliminary report on effects of photocoagulation therapy. Am J Ophthalmol. Vol. 81, pp. 383-396.

Ziemer DC, Kolm p, Weintraub WS. et al. (2010) Glucose independent, black-white differences in hemoglobin A1c levels: a cross sectional analysis of 2 studies. Ann Intern Med. Vol. 153, pp. 770-777. 